\title{
Primary Hepatic Osteosarcoma
}

\author{
Atta Nawabi • Sidhartha Rath • Nicholas Nissen • \\ Charles Forscher • Steven Colquhoun • Joseph Lee • \\ Stephen Geller • Anna Wong • Andrew S. Klein
}

Received: 30 September 2008 / Accepted: 26 February 2009/Published online: 18 March 2009

(C) 2009 The Author(s). This article is published with open access at Springerlink.com

Keywords Osteosarcoma $\cdot$ Hepatic neoplasm .

Hepatic malignancy

Extraskeletal osteosarcoma (EOS) is a rare entity. Most human cases have been described in the soft tissues of the limb. ${ }^{1}$ Primary hepatic osteosarcoma is extremely uncommon with only seven cases reported in the world's literature. Of these cases, no patient survived more than 8 weeks from the time of diagnosis. We describe a young patient with a symptomatic primary hepatic osteosarcoma that was successfully treated by surgical resection and adjuvant chemotherapy. She is alive and tumor free 3 years after surgery.

\section{Case Report}

A healthy, athletic 19-year-old African American woman presented for a surgical evaluation of a newly diagnosed hepatic mass. The patient reported approximately 18 months of occasional and intermittent episodes of epigastric discomfort. Recently, she had developed symptoms of early satiety and anorexia. On physical exam, she had vague diffuse upper abdominal tenderness and a firm, palpable mass in the left upper quadrant extending to the midline. A calcified left upper quadrant mass was seen on abdominal $\mathrm{X}$-ray taken during an emergency room visit. Laboratory

A. Nawabi $\cdot$ S. Rath $\cdot$ N. Nissen $\cdot$ C. Forscher $\cdot$ S. Colquhoun •

J. Lee $\cdot$ S. Geller $\cdot$ A. Wong $\cdot$ A. S. Klein $(\bowtie)$

Department of Surgery, Cedars-Sinai Medical Center,

8635 W. 3rd Street, 590 West,

Los Angeles, CA 90048, USA

e-mail: kleinas@cshs.org evaluation was remarkable for moderate elevations of alkaline phosphatase and alpha fetoprotein.

To further evaluate the mass, a CT scan of the chest, abdomen, and pelvis was performed (Fig. 1), which showed a large heterogeneous mass occupying the left lobe of the liver with a $5-\mathrm{cm}$ area of calcification in the anterior aspect. MRI demonstrated a single heterogeneously enhancing $15 \times$ $10 \times 13.8 \mathrm{~cm}$ lobulated mass inseparable from the left lobe of the liver (Fig. 2). The left portal vein was not visualized and there was no biliary dilatation. T1- and T2-weighted sequences showed a $5-\mathrm{cm}$ area of signal hypointensity with multiple hypointense non-enhancing linear areas throughout the mass consistent with areas of calcification. A small amount of intraperitoneal free fluid was present. Staging chest and brain CT demonstrated no evidence for metastatic disease. Whole body Tc-99m MDP bone scan demonstrated no evidence of skeletal metastatic disease. The radiologic differential diagnosis includes sarcoma, including metastatic osteosarcoma, and fibrolamellar hepatocellular carcinoma.

At operation, a large, scarred lesion replacing the left hepatic lobe was immediately identified. The lesion extended across the falciform ligament into segment 4 of the liver. It was also adherent to, but did not invade, the stomach. The abdominal cavity was examined and no metastatic deposits were noted. The right hepatic lobe was examined with both palpation and intraoperative ultrasound, and was free of tumor. A left hepatic lobectomy was performed with en-bloc cholecystectomy using a combination of electrocautery and hydrojet dissection. The middle hepatic vein was not involved in the mass and was left insitu. Her post-operative recovery was uneventful. She was discharged on the fifth post-operative day. 


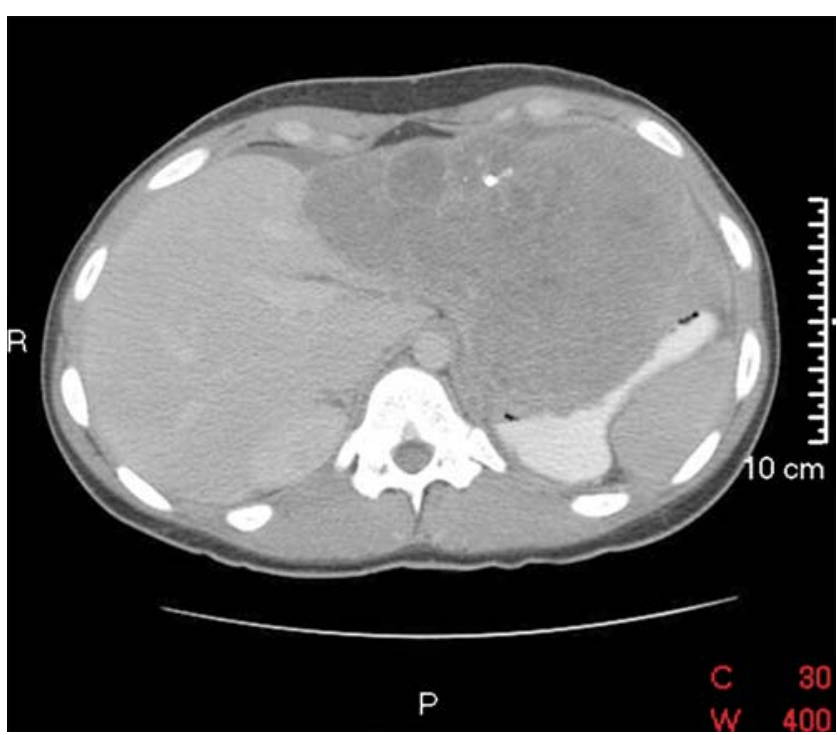

Figure 1 Post-contrast axial CT demonstrates a heterogeneous mass replacing the left lobe of the liver, hypoenhancing to liver parenchyma, with areas of calcification. It displaces the stomach posteriorly and inferiorly. The left portal vein is not visualized.

The patient received adjuvant chemotherapy with ifosfamide and mesna at $14 \mathrm{~g} / \mathrm{m}^{2}$ for two courses followed by cisplatin at $100 \mathrm{mg} / \mathrm{m}^{2}$ with doxorubicin at $75 \mathrm{mg} / \mathrm{m}^{2}$. Chemotherapy courses were administered at 3-week intervals. She received a total of four courses of ifosfamide and two courses of cisplatin and doxorubicin with a cumulative dose of doxorubicin of $150 \mathrm{mg} / \mathrm{m}^{2}$. The patient has been followed clinically as well as by serial CT imaging of her

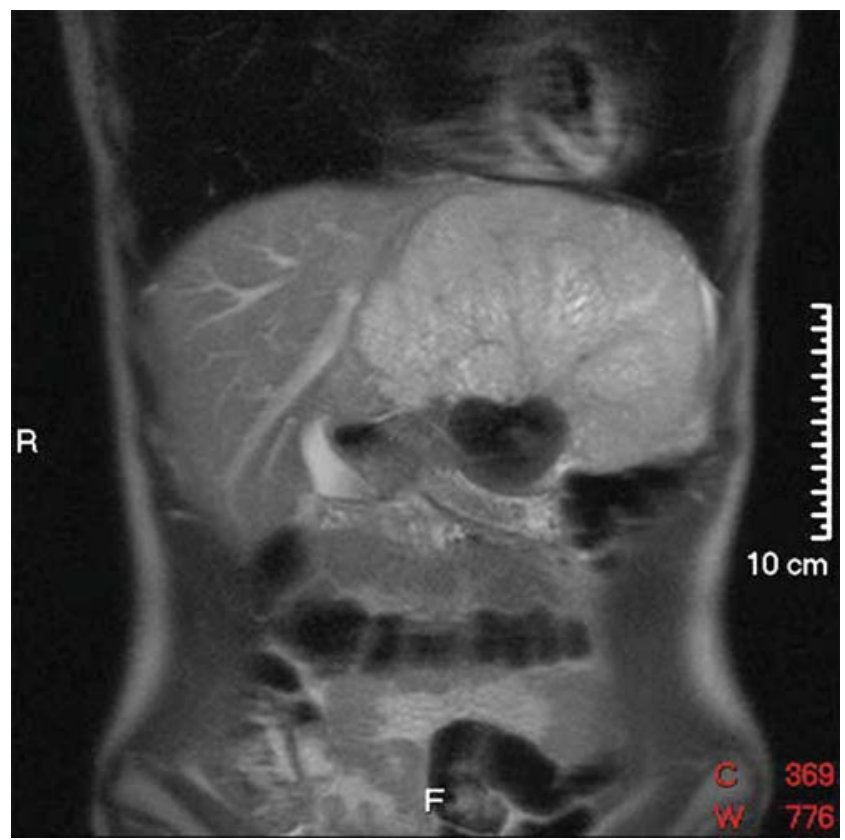

Figure 2 Coronal T2-weighted MR demonstrating the lobulated mass, predominantly hyperintense to liver. The calcified component is hypointense.

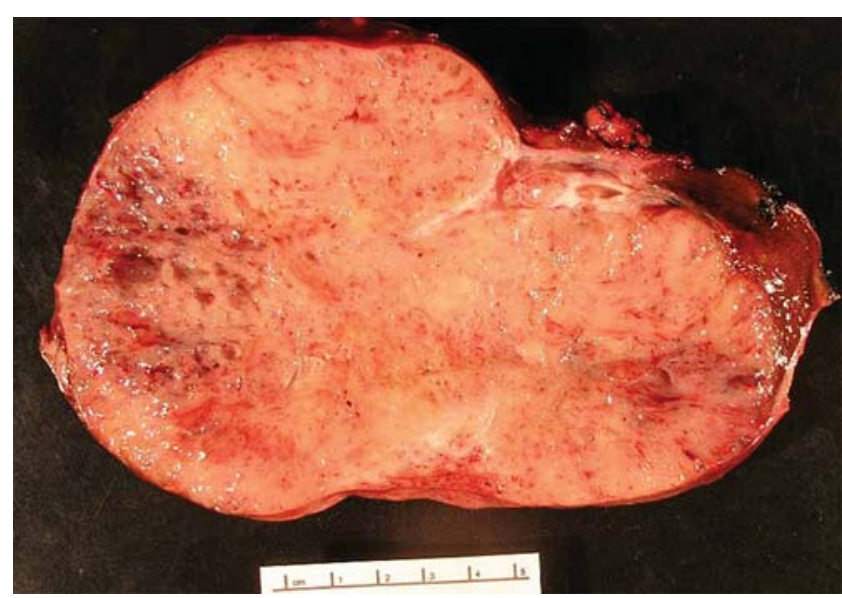

Figure 3 Cut surface of the resection specimen shows a tan-gray, rubbery, firm well-demarcated tumor with multifocal areas of hemorrhage and a thrombus at the right upper portion of the specimen. The scale shows centimeters.

abdomen, pelvis, and chest. She is alive without recurrence 36 months after surgery.

Pathologic examination of the left lobe resection revealed a $15.0 \times 13.8 \times 12.0 \mathrm{~cm}, 1,390 \mathrm{~g}$ well-demarcated non-encapsulated tan-gray rubbery intraparenchymal mass with diffuse punctate hemorrhagic areas involving $95 \%$ of the specimen (Fig. 3). A focal $5.5 \times 4.5 \times 4.5 \mathrm{~cm}$ gray-white calcified area was present and a thrombus was in a large portal vein. The surrounding liver parenchyma was tanbrown and non-cirrhotic appearing. The specimen was processed with formalin fixation and paraffin embedding.

Histological examination showed an undifferentiated spindle cell neoplasm with foci of osteoid formation. The calcified area was composed of multiple osteoid islands circumferentially surrounded by undifferentiated malignant spindle cells. These pleomorphic spindle cells were characterized by indistinct cytoplasmic borders, vesicular

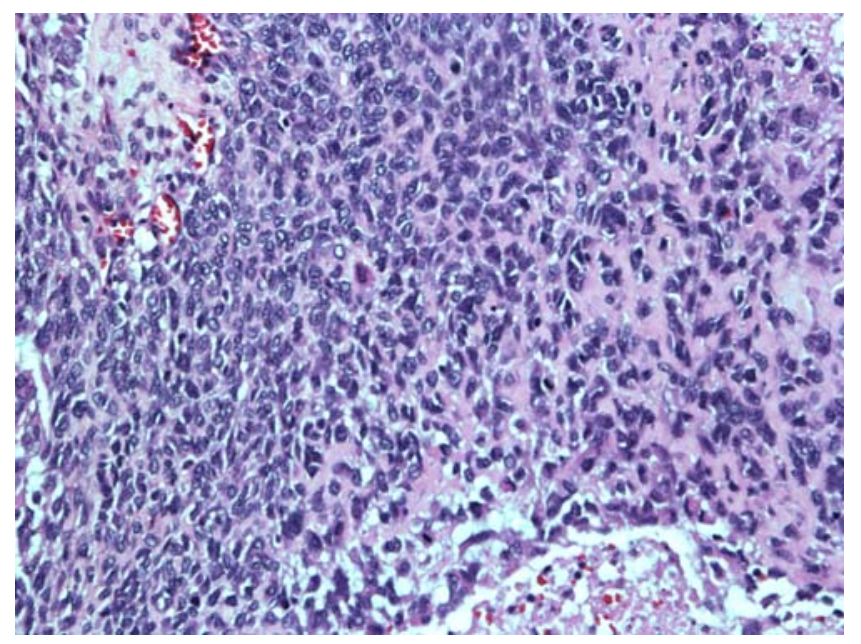

Figure 4 Malignant spindle cells with high mitotic activity, lacey osteoid formation, and necrosis $(\mathrm{H} \& \mathrm{E} \times 20)$. 
nuclei with inconspicuous nucleoli, and irregular nuclear membrane (Fig. 4). The spindle cells reacted with antibody directed against vimentin and there was no immunostaining for smooth muscle actin (SMA), S-100, desmin, epithelial membrane antigen (EMA), CD 57, or AE1/3. A high mitotic rate with greater than $80 \%$ staining for proliferative marker Ki-67 was seen. Tumor was present in the portal vein and in many intra-hepatic portal vein branches.

\section{Discussion}

Skeletal osteosarcoma is the most common malignant bone tumor in children and adolescents. Modern multimodality therapies including surgery and multiagent chemotherapy have produced $60-70 \% 3$-year survival rates. ${ }^{2}$ Extraskeletal osteosarcoma is a rare entity and case descriptions can be found throughout the literature mostly in the limbs and limb girdles. ${ }^{3}$ Even fewer are reports of parenchymal osteosarcoma, but such cases have been documented in thyroid, kidney, gallbladder, breast, mesentery, liver, and colon. ${ }^{4-10}$

In adults, primary sarcomas of the liver are very uncommon. Attention has been focused particularly on angiosarcoma in connection with thorotrast and polyvinyl chloride. ${ }^{11}$ Cases of primary hepatic fibrosarcoma and leiomyosarcoma have been reported as have undifferentiated sarcomas, though these tumors are seen almost exclusively in the pediatric age group. ${ }^{12}$ Primary osteosarcoma of the liver is an exceedingly rare neoplasm of the liver which requires that the presence of other neoplastic components be excluded..$^{13}$ It is important to note that, after extensive radiologic imaging and physical examination, no evidence of a primary skeletal osteosarcoma was found in the patient described in this report. Sumiyoshi and Niho reported the case of a 52-year-old man who was hospitalized for hepatic failure. ${ }^{14} \mathrm{He}$ died 2 months after onset of symptoms. Autopsy revealed a cirrhotic liver with a large mass with histologic features of osteosarcoma. A literature search revealed a total of seven cases of primary osteosarcoma of the liver in humans and two case reports in animals. ${ }^{8,13-20}$ We are unaware of a pre-operative diagnosis being made on any patient with this disease. This may be related to the rarity of the lesion or, alternatively, that if a biopsy were to return osteoid in a large liver tumor, various more common tumors would be suspected including hepatoblastoma, hepatic teratoma, malignant mesenchymoma, carcinosarcoma, hepatic angiosarcoma, or other hepatic sarcoma. Moreover, when mixed epithelial components are present, the pattern of differentiation is essential to characterize the tumor. This would be impossible with only a needle biopsy.

The age of patients with primary hepatic osteosarcoma in prior reports ranged from 52 to 73 years. Each previously described case resulted in either quick progression to death after diagnosis or the diagnosis was only established at autopsy. This case is notable for the young age at presentation (19 years old) and the prolonged disease-free survival she has experienced following surgery and adjuvant chemotherapy with multiagent chemotherapy.

Open Access This article is distributed under the terms of the Creative Commons Attribution Noncommercial License which permits any noncommercial use, distribution, and reproduction in any medium, provided the original author(s) and source are credited.

\section{References}

1. Allan CJ, Soule EH. Osteogenic sarcoma of the somatic soft tissues: clinicopathologic study of 26 cases and review of literature. Cancer 1971;27(5):1121-1133. doi:10.1002/1097-0142(197105)27:5<1121:: AID-CNCR2820270519>3.0.CO;2-3.

2. Marina N, Gebhardt M, Teot L, et al. Biology and therapeutic advances for pediatric osteosarcoma. Oncologist 2004;9:422-441. doi:10.1634/theoncologist.9-4-422.

3. Bane BL, Evans HL, Ro JY, et al. Extraskeletal osteosarcoma, a clinicopathologic review of 26 cases. Cancer 1990;65:2762-2770. doi:10.1002/1097-0142(19900615)65:12<2762::AIDCNCR2820651226>3.0.CO;2-K.

4. Momoi H, Wada Y, Sarumaru S, et al. Primary osteosarcoma of the breast. Breast Cancer 2004;11(4):396-400. doi:10.1007/ BF02968048.

5. Olgyai G, Horváth V, Kocsis J, et al. Extraskeletal osteosarcoma in the gallbladder. Magy Seb 2003;56(2):57-60.

6. Choudur HN, Munk PL, Nielson TO, Ryan AG. Primary mesenteric extraskeletal osteosarcoma in the pelvic cavity. Skeletal Radiol 2005;34(10):649-652. doi:10.1007/s00256-0050909-8.

7. Shimazu K, Funata N, Yamamoto Y, Mori T. Primary osteosarcoma arising in the colon: report of a case. Dis Colon Rectum 2001;44(9):1367-1370. doi:10.1007/BF02234799.

8. Govender D, Raghubar KN. Primary hepatic osteosarcoma: case report and literature review. Pathology 1998;30:323-325. doi:10.1080/00313029800169556.

9. Trowell JE, Arkell DG. Osteosarcoma of thyroid gland. J Pathol 1976;119:123-127. doi:10.1002/path.1711190208.

10. Axelrod R, Naidech HJ, Myers J, Steinberg A. Primary osteosarcoma of the kidney. Cancer 1978;41(2):724-727. doi:10.1002/ 1097-0142(197802)41:2<724::AID-CNCR2820410244>3.0. $\mathrm{CO} ; 2-\mathrm{N}$.

11. Ishak KG. Mesenchymal tumors of the liver. In Okuda K, Peters RL, eds. Hepatocellular carcinoma. New York: Wiley, 1976, pp 247-307.

12. Stocker JT, Ishak KG. Undifferentiated (embryonal) sarcoma of the liver: report of 31 cases. Cancer 1978;42:336-348. doi:10.1002/10970142(197807)42:1<336::AID-CNCR2820420151>3.0.CO;2-V.

13. Von Hochstetter AR, Hättenschwiler J, Vogt M. Primary osteosarcoma of the liver. Cancer 1987;60:2312-2317. doi:10.1002/ 1097-0142(19871101)60:9<2312::AID-CNCR2820600933>3.0. CO;2-W.

14. Sumiyoshi A, Niho Y. Primary osteogenic sarcoma of the liver: report of an autopsy case. Acta Pathol Jpn 1971;21:305-312. 
15. Boldt C, Pabst U, Nitsche R, Bürrig KF. Primary osteosarcoma of the liver. Case report and literature review. Pathologe 1999;20 (6):359-364. doi:10.1007/s002920050372.

16. Kitayama Y, Sugimura H, Arai T, Nagamatsu K, Kino I. Primary osteosarcoma arising from cirrhotic liver. Pathol Int 1995;45 (4):320-325. doi:10.1111/j.1440-1827.1995.tb03464.x.

17. Liony C, Lemarchand P, Manchon ND, et al. A case of primary osteosarcoma of the liver. Gastroenterol Clin Biol 1990;14 (12):1003-1006.
18. Hatori M, Hosaka M, Watanabe $\mathrm{M}$, et al. Osteosarcoma in a patient with neurofibromatosis type 1: a case report and review of the literature. Tohoku J Exp Med 2006;208(4):343-348. doi:10.1620/tjem.208.343.

19. Patnaik AK, Kuy SK, Johnson JF. Extraskeletal osteosarcoma of the liver in a dog. J Small Anim Pract 1976;17:365-370. doi:10.1111/j.1748-5827.1976.tb06972.x.

20. Jeraj K, Yano B, Osborn CA. Primary hepatic osteosarcoma in dog. J Am Vet Med Assoc 1981;179:1000-1003. 